\title{
Rationally designed BCL6 inhibitors target activated B cell diffuse large B cell lymphoma
}

\author{
Mariano G. Cardenas, ${ }^{1}$ Wenbo Yu, ${ }^{2}$ Wendy Beguelin, ${ }^{1}$ Matthew R. Teater, ${ }^{1}$ Huimin Geng, ${ }^{1,3}$ Rebecca L. Goldstein, ${ }^{1}$ Erin Oswald, \\ Katerina Hatzi, ${ }^{1}$ Shao-Ning Yang, ${ }^{1}$ Joanna Cohen, ${ }^{1}$ Rita Shaknovich, ${ }^{1}$ Kenno Vanommeslaeghe, ${ }^{2,4}$ Huimin Cheng, ${ }^{2}$ \\ Dongdong Liang, ${ }^{2}$ Hyo Je Cho, ${ }^{5}$ Joshua Abbott, ${ }^{5}$ Wayne Tam, ${ }^{6}$ Wei Du, John P. Leonard, ${ }^{1}$ Olivier Elemento, ${ }^{7}$ \\ Leandro Cerchietti, ${ }^{1}$ Tomasz Cierpicki, ${ }^{5}$ Fengtian Xue, ${ }^{2}$ Alexander D. MacKerell Jr., ${ }^{2}$ and Ari M. Melnick ${ }^{1}$ \\ 'Weill Cornell Medical College, Department of Hematology/Oncology, New York, New York, USA. ²Computer-Aided Drug Design Center, University of Maryland, School of Pharmacy, \\ Department of Pharmaceutical Sciences, Baltimore, Maryland, USA. ${ }^{3}$ UCSF, Department of Laboratory Medicine, San Francisco, California, USA. ${ }^{4}$ Department of Analytical Chemistry and \\ Pharmaceutical Technology (FABI), Center for Pharmaceutical Research, Vrije Universiteit Brussel, Brussels, Belgium. ${ }^{5}$ University of Michigan, Department of Pathology, Ann Arbor, Michigan, USA. \\ ${ }^{6}$ Department of Pathology and Laboratory Medicine and ${ }^{7}$ Department of Physiology and Biophysics, Weill Cornell Medical College, New York, New York, USA.
}

Diffuse large B cell lymphomas (DLBCLs) arise from proliferating B cells transiting different stages of the germinal center reaction. In activated B cell DLBCLs (ABC-DLBCLs), a class of DLBCLs that respond poorly to current therapies, chromosomal translocations and amplification lead to constitutive expression of the B cell lymphoma 6 (BCL6) oncogene. The role of BCL6 in maintaining these lymphomas has not been investigated. Here, we designed small-molecule inhibitors that display higher affinity for BCL6 than its endogenous corepressor ligands to evaluate their therapeutic efficacy for targeting ABC-DLBCL. We used an in silico drug design functional-group mapping approach called SILCS to create a specific BCL6 inhibitor called FX1 that has 10 -fold greater potency than endogenous corepressors and binds an essential region of the BCL6 lateral groove. FX1 disrupted formation of the BCL6 repression complex, reactivated BCL6 target genes, and mimicked the phenotype of mice engineered to express BCL6 with corepressor binding site mutations. Low doses of FX1 induced regression of established tumors in mice bearing DLBCL xenografts. Furthermore, FX1 suppressed ABC-DLBCL cells in vitro and in vivo, as well as primary human $A B C-D L B C L$ specimens ex vivo. These findings indicate that ABC-DLBCL is a BCL6-dependent disease that can be targeted by rationally designed inhibitors that exceed the binding affinity of natural BCL6 ligands.

\section{Introduction}

Diffuse large B cell lymphomas (DLBCLs) are the most common lymphoid neoplasms (1). Most DLBCLs arise from B cells that have transited through the germinal center (GC) reaction, a mutagenic process wherein B cells undergo rapid proliferation and Ig somatic hypermutation $(2,3)$. DLBCLs are generally classified according to whether their gene expression profiles are more closely related to germinal center B cells (GCB-DLBCL), or cells that are exiting the $\mathrm{GC}$ reaction and initiating plasma cell differentiation (the activated $\mathrm{B}$ cell, or ABC-DLBCL, subtype) $(4,5)$. ABC-DLBCLs characteristically exhibit unfavorable clinical outcome as compared with GCB-DLBCLs $(4,5)$. The standard therapy for treatment of DLBCL consists of a combination of CD20 mAbs with a multiagent chemotherapy regimen called CHOP (6-9). Even when suc-

Authorship note: M.C. Cardenas and W. Yu are co-first authors.

Conflict of interest: A.D. MacKerell Jr. is co-founder and Chief Science Officer of SilcsBio LLC. A.M. Melnick is a consultant for Boehringer Ingelheim GmbH, Eli Lilly and Co., Epizyme Inc., Janssen Pharmaceuticals Inc., and Hoffmann-La Roche Ltd. A.M. Melnick receives research funding from Eli Lilly, Janssen, and Roche. These consulting and research interactions are in no way related to the current article. There was no interaction with any of these companies on this project. A.M. Melnick, L. Cerchietti, M.G. Cardenas,

F. Xue, and A.D. MacKerell are inventors of the patent PCT/US14/42556 "BCL6 inhibitors as anticancer agents."

Submitted: January 4, 2016; Accepted: June 3, 2016.

Reference information: J Clin Invest. 2016;126(9):3351-3362. doi:10.1172/JCI85795. cessful, this regimen results in significant toxicity, and over $40 \%$ of patients relapse and die of their disease (6-9). Novel therapies are needed both to improve the outcome for ABC-DLBCL patients and to reduce toxicity of current regimens.

One of the hallmarks of ABC-DLBCL are translocations and amplifications of the B cell lymphoma 6 (BCL6) locus (10-12). BCL6 is a transcriptional repressor required for B cells to form GCs during the humoral immune response (13-15). BCL6 is downregulated as B cells exit the GC reaction and initiate plasma cell differentiation (2). BCL6 enables proliferation and survival of GC B cells by repressing various checkpoint pathways, and blocks plasma cell differentiation by directly repressing IRF 4 and $P R D M 1$, genes that drive sequential steps in GC exit and terminal differentiation (2, 3). The BCL6 translocations and amplifications occurring in ABCDLBCLs presumably have the effect of aberrantly maintaining BCL6 expression. This could drive lymphomagenesis by sustaining growth and survival of mutating B cells and preventing their further differentiation. Indeed, constitutive expression of BCL6 in mouse GC B cells results in the development of DLBCL $(16,17)$. However, because ABC-DLBCLs often express lower levels of BCL6 than GCB-DLBCLs (4), a potential role for BCL6 in maintaining these lymphomas has not been investigated.

BCL6 represses genes that would otherwise prevent the proliferation and survival of GC B cells by recruiting the SMRT, NCOR, and BCOR corepressors to its $N$-terminal BTB domain $(18,19)$. 
The binding site for these corepressors consists of an extended groove formed through obligate homodimerization of BCL6 BTB domains $(20,21)$. Mice that express a form of BCL6 with mutation in the corepressor-binding groove fail to form GCs (22). Decoy peptides that contain the SMRT corepressor fragment that binds to the BCL6 BTB domain and compete with endogenous corepressors also disrupt GC formation when administered to immunized mice $(23,24)$. These same peptides were also shown to kill DLBCL cells $(23,24)$. These data led to the notion of therapeutic targeting of the BCL6 BTB domain lateral groove as a potential therapy for B cell lymphomas. Proof-of-principle studies supporting this strategy involved the development of the peptidomimetic drug RI-BPI (24), and small-molecule inhibitors of BCL6 such as compound 79-6 (25). However, all of these compounds bind to BCL6 with significantly weaker affinity than endogenous corepressor proteins, which could potentially limit their utility. Herein, we used a novel in silico chemical functional group-based screening approach called SILCS $(26,27)$ to design compounds that could bind to the BCL6 lateral groove with higher affinity than endogenous corepressors and yet retain specificity for BCL6. The gain in smallmolecule affinity proves that BCL6 is a druggable target in spite of the extended nature of its protein interaction surface. Using these new compounds and BCL6 shRNA, we show that ABC-DLBCLs are biologically dependent on BCL6 and that BCL6-targeted therapy represents a compelling new strategy for the treatment of these aggressive tumors.

\section{Results}

SILCS-guided design of BCL6 inhibitor compounds. The relevance of previously developed BCL6 inhibitors is restricted by their low binding affinity to the BCL6 BTB domain groove motif. To overcome this limitation and identify superior inhibitors, we applied a method called SILCS that generates 3D probability binding distributions of chemical functional groups on protein surfaces, information that may be used to direct ligand design (Figure 1A). These "FragMaps" include information on protein flexibility, proteinligand interactions, and protein and ligand desolvation. The latter is particularly significant with BCL6 as it allows determination of the importance of negative (e.g., carboxylate) versus neutral hydrogen bond acceptors that were important for the binding of first-generation BCL6 inhibitors (25).

As a first step to SILCS-guided design of new molecules, we overlaid the most active first-generation BCL6 inhibitor, 79-6 (IC ${ }_{50} \sim 200 \mu \mathrm{M}$; ref. 25), with the BCL6 BTB domain SILCS FragMap (Figure 1B). This analysis revealed (a) overlap of FragMap aromatic and aliphatic moieties with the indolin-2-one ring of 79-6, underlining the importance of maintaining the aromatic ring including hydrophobic substituents to occupy this site; (b) overlap of one of the two 79-6 carboxylate groups with a negative acceptor FragMap, showing that only 1 of the 2 carboxylate groups is necessary to form the key charged interaction with Arg-28; and (c) overlap of the carbonyl and the $\mathrm{NH}$ of the indoline-2-one ring with neutral acceptors and donor FragMaps, further supporting the rationale for maintaining the indoline-2-one ring in the design of new compounds. In addition, the lack of FragMap overlap with the 79-6 rhodanine moiety suggested that it does not make a dominant contribution to binding. This motivated design of a subset of analogs in which the exocyclic sulfur of the rhodanine is replaced with an oxygen and conjugation with the indoline-2-one ring is no longer present. Alteration of the rhodanine also has the advantage of avoiding this potentially promiscuous, pan-assay interference compounds (PAINS) (28) moiety, although our previous experimental analysis of 79-6 indicated that the presence of this moiety is not problematic, such that its omission was not considered essential. Collectively, these data define an aromatic pocket and an acid pocket as optimal features on which to base design of improved small-molecule binders to the BTB domain.

Based on the above observations we focused our design effort on functionalization of the indoline-2-one ring and variation of the length of the linker to acid group. Once a series of synthetically accessible ligands were designed, they were subjected to quantitative ranking using Monte Carlo-SILCS (MC-SILCS) docking, from which the ligand grid free energy (LGFE), an estimate of the binding affinity of a compound $(26,27)$, was determined. These compounds were subsequently synthesized and purified for experimental assay. The compounds along with their LGFE scores are shown in Supplemental Figure 1, A and B (supplemental material available online with this article; doi:10.1172/JCI85795DS1).

Compound activity was then screened using a BCL6 ${ }^{\text {BTB }}$-GAL4 DNA binding domain luciferase reporter assay, which measures potency on the basis of reversal of BCL6-mediated transcriptional repression (25) (Supplemental Figure 1B). Among the tested molecules, FX1 was identified as the most active and selective BCL6 BTB inhibitor. Consistent with this activity, FX1 was also 1 of the top-ranked compounds on the basis of MC-SILCS LGFE scores (Supplemental Figure 1B). The predicted binding orientation of FX1 was obtained using MC-SILCS docking, shown in Figure 1C overlaid with the SILCS FragMaps as well as the established binding configuration of 79-6. The similar orientations of FX1 and 79-6 support the role of the indolin-2-one ring along with its halogen substituent in binding, along with the need for only 1 carboxylate group to form charge-charge interaction with the acid site associated with the side chain of Arg-28 (Figure 1C). Given its optimized structure and improved docking, it was predicted that this new compound would be a more effective BCL6 inhibitor than the previously published 79-6 (25).

FX1 is specific to BCL6 and binds with a greater affinity than the natural BCL6 ligand SMRT. Microscale thermophoresis (MST) $(29,30)$ was used to compare and contrast the relative BCL6 BTB domain binding affinities of FX1, 79-6, and the natural BCL6 binding domain (BBD) from SMRT. The dissociation constants $\left(K_{\mathrm{D}}\right)$ of these interactions were determined after incubation of $200 \mathrm{nM}$ of fluorescently labeled BCL6 BTB domain with different concentrations of SMRT BBD, 79-6, or FX1 for 10 minutes (Figure 1D). The SMRT BBD exhibited a $K_{\mathrm{D}}$ of $30 \pm 3 \mu \mathrm{M}$, consistent with previously published results $(20,23)$. Remarkably, FX1 bound with a more than 4 -fold greater affinity than SMRT $\left(K_{\mathrm{D}}\right.$ of $\left.7 \pm 3 \mu \mathrm{M}\right)$, whereas 79-6 was considerably weaker than SMRT ( $K_{\mathrm{D}}$ of $\left.129 \pm 25 \mu \mathrm{M}\right)$.

The BCL6 BTB domain lateral groove is not conserved in other BTB domain proteins, providing the basis for development of compounds selective for BCL6 (20). Hence, to determine whether FX1 and related compounds might disrupt the repressor activity of related transcription factors, we performed additional reporter assays using the BTB domains from HIC1, PLZF, and Kaiso (Sup- 
A

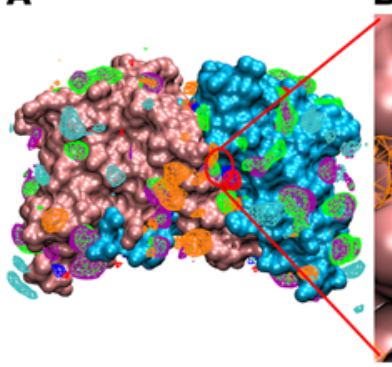

D

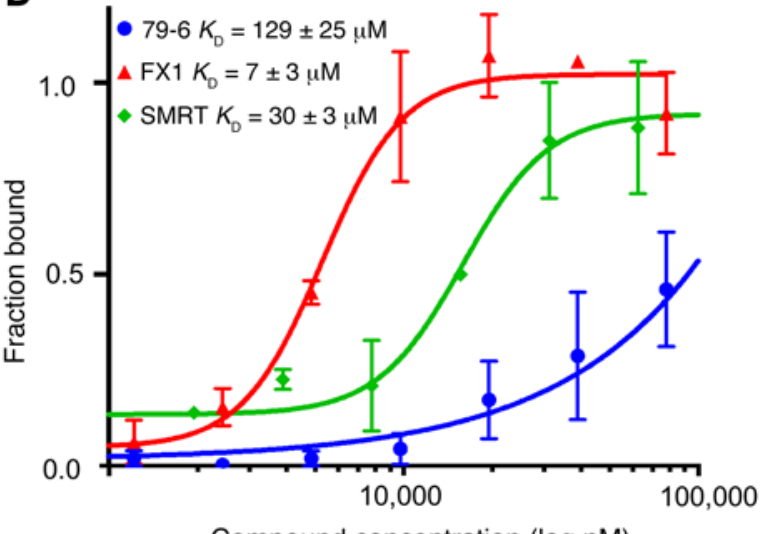

Compound concentration $(\log \mathrm{nM})$

F

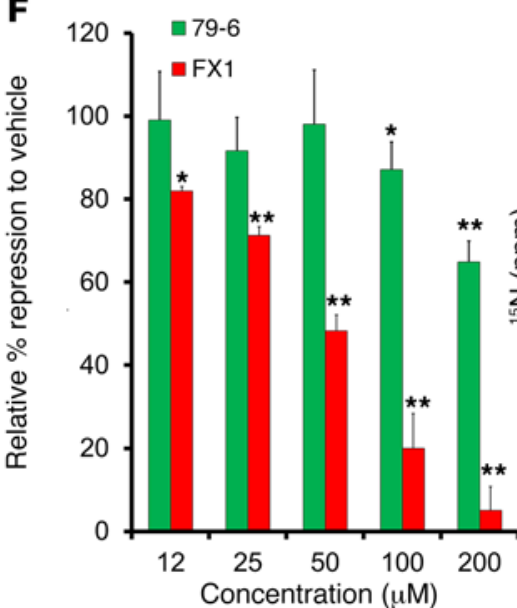

B

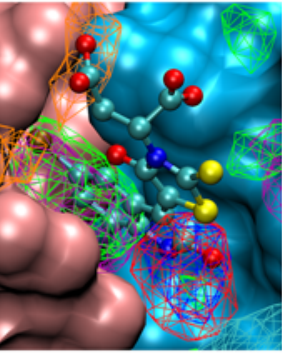

E
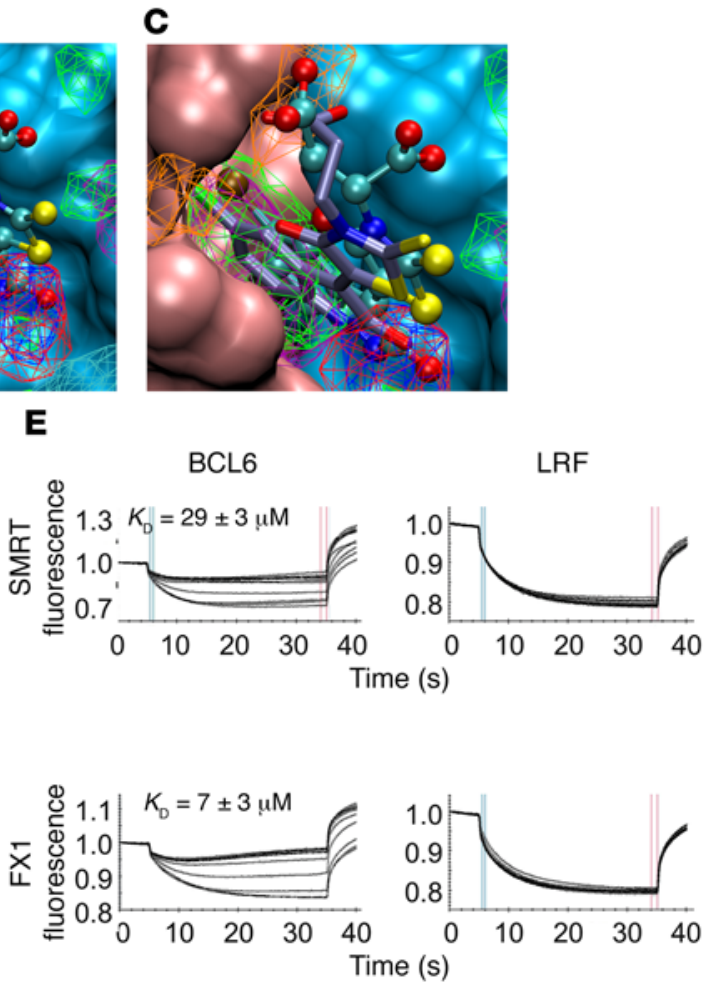

H
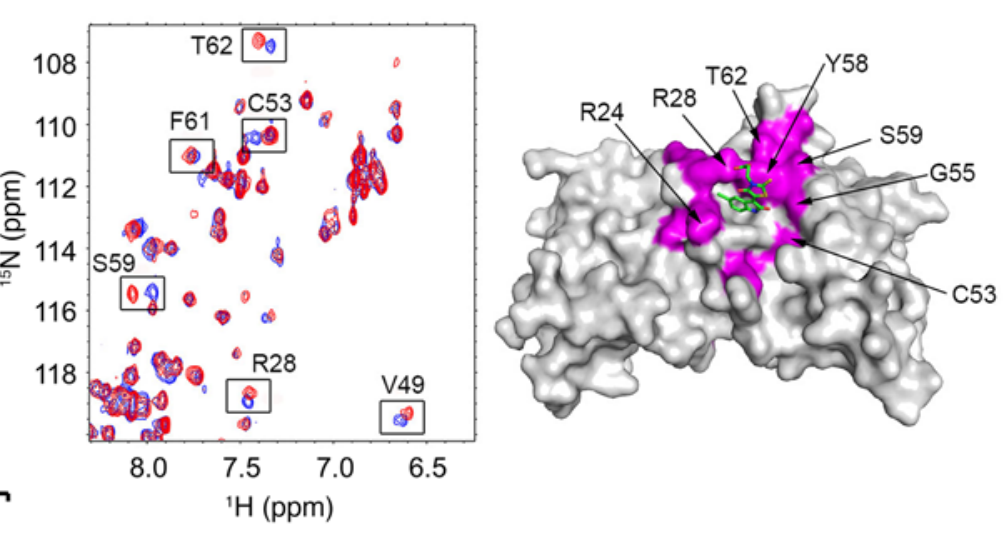

Figure 1. Identification and characterization of FX1 as a BCL6 BTB inhibitor. (A) SILCS FragMaps overlaid on the apo BCL6 BTB domain with the aromatic (purple), aliphatic (green), hydrogen bond donor (blue) and acceptor (red), and charged acceptor (orange) maps. (B and C) Superposition of the SILCS FragMaps with 79-6 alone (B) and with FX1 and 79-6 in complex with BCL6 BTB (C). (D) Comparison of the affinity of the natural ligand SMRT peptide and the small molecules 79-6 and FX1 to BCL6 BTB domains determined by microscale thermophoresis (MST; $n=3$ independent experiments; error bars represent the SD). (E) Comparison of the MST results of SMRT and FX1 interaction with the BTB domains of BCL6 or LRF. Results represent mean \pm SD of 3 independent experiments. (F) Luciferase reporter assays showing activity of 79-6 or FX1 as compared with vehicle against the repressor activity of a GAL4 ${ }^{\mathrm{DBD}}$ $\mathrm{BCL6}^{\mathrm{BTB}}$ fusion construct compared with the $\mathrm{CAL} 4^{\mathrm{DBD}}$ alone. The $y$ axis represents the relative percentage of repression mediated by the fusion protein in the presence of vehicle, set as $100 \%$. Bars represent mean \pm SD of 3 independent experiments. $\left({ }^{*} P<0.05,{ }^{* *} P<0.005, t\right.$ test.) (G) Heteronuclear single quantum coherence spectrum of $250 \mu$ M BCL6 BTB with 5\% DMSO (red) is superimposed onto the spectrum of BTB with $500 \mu$ M FX1 (blue). Residues that experience the most significant chemical shift perturbation are labeled. (H) A graphical representation of the BCL6 BTB domain homodimer based on Protein Data Bank (PDB) structure 1R2B is shown, indicating residues perturbed upon binding of FX1 in magenta.

plemental Figure 1B). Almost none of the compounds (including FX1) significantly affected transcriptional repression by these proteins. We next tested whether FX1 could bind to the closely related BTB domain from the LRF (BTB7A) transcription factor in MST assays. Neither SMRT nor FX1 could bind to the LRF BTB domain (Figure 1E), confirming that FX1 binding is selective to
BCL6. Consistent with its greater binding affinity versus 79-6, FX1 exhibited 10-fold greater inhibitory activity against the BCL6 BTB domain in reporter assays $\left(\mathrm{FX1} \mathrm{IC}_{50} \sim 35 \mu \mathrm{M}\right.$ vs. $79-6 \mathrm{IC}_{50} \sim 318 \mu \mathrm{M}$; Figure 1F). To further explore compound selectivity, we measured the activity of FX1 against a panel of 50 different kinases. These experiments were performed at an FX1 concentration that 
A

B

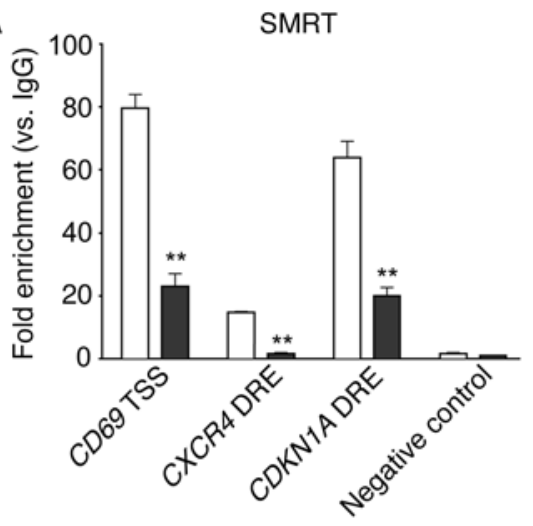

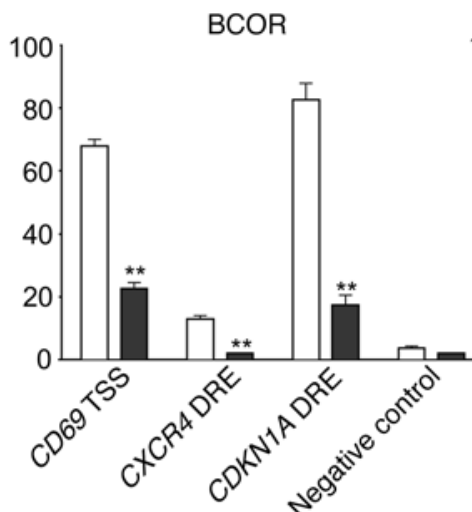

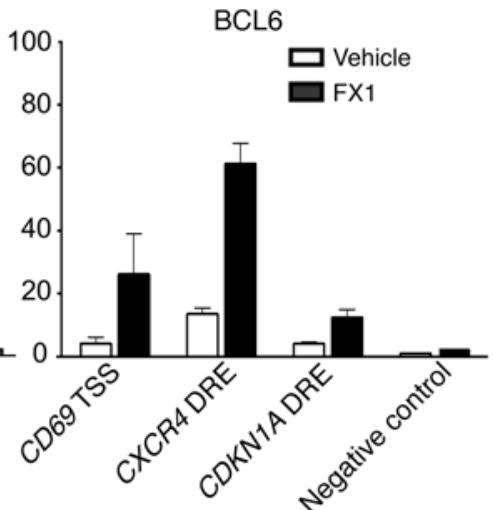

SUDHL-6

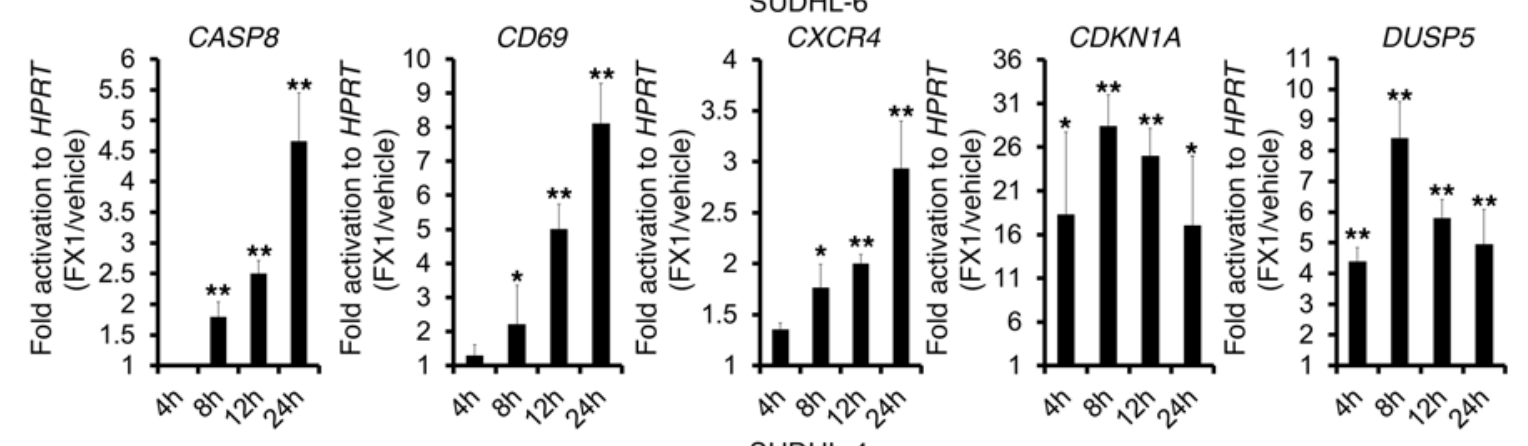

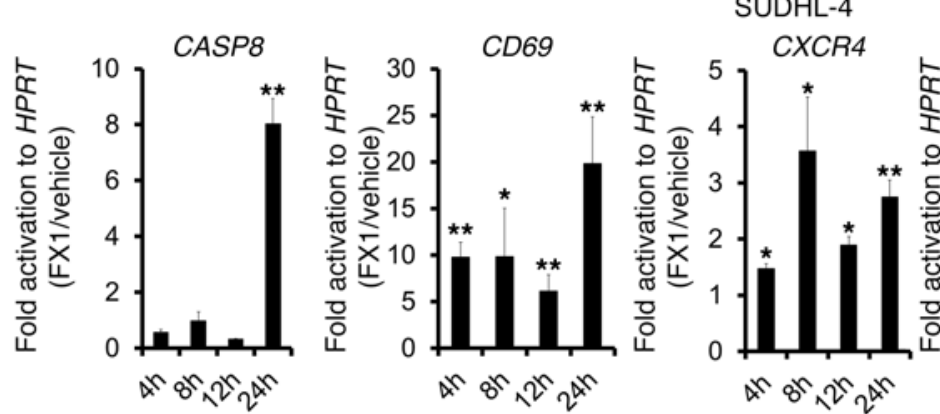

C SUDHL-4 upregulated genes

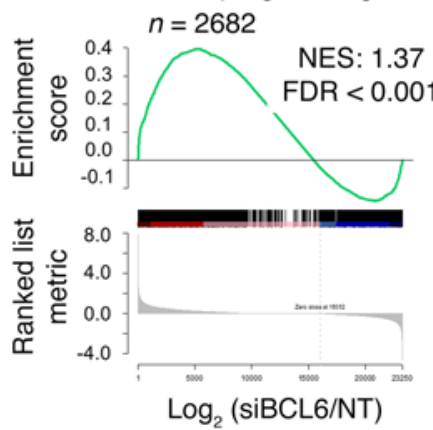

SUDHL-6 upregulated genes

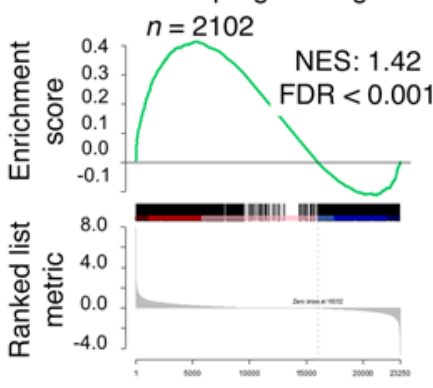

$\log _{2}(\operatorname{siBCL6/NT})$

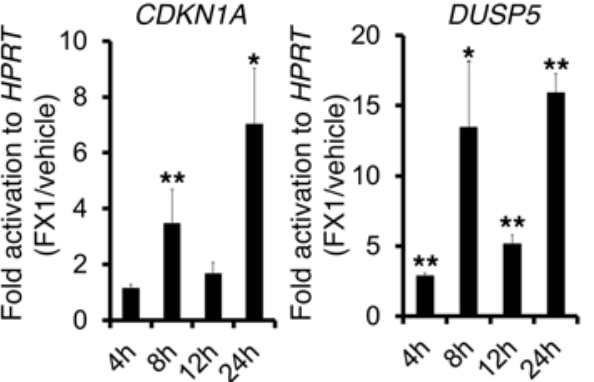

Farage upregulated genes

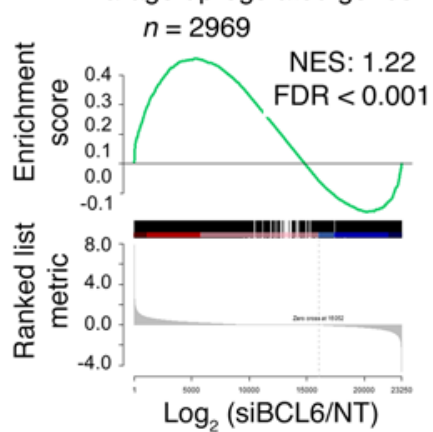

Figure 2. FX1 disrupts BCL6 repression complexes and induces derepression of BCL6 target genes. (A) Quantitative ChIP was performed in SUDHL-6 cells exposed to FX1 (black bars) or vehicle (white bars) in DLBCL cells using antibodies for BCL6, SMRT, BCOR, or lgG control to enrich for known BCL6 binding sites in the CD69, CXCR4, and DUSP5 loci, or a negative control region. The $y$ axis represents fold enrichment of binding versus input, as compared with IgC control (**P<0.005, $t$ test). (B) Quantitative PCR was performed in SUDHL-6 and SUDHL-4 cells after exposure to FX1 or vehicle to measure abundance of the BCL6 target genes CASP8, CD69, CXCR4, CDKN1A, and DUSP5. The $y$ axis shows fold enrichment versus HPRT mRNA based on the $\triangle \Delta^{\text {ct }}$ values $\left({ }^{*} P<0.05,{ }^{* *} P<0.005, t\right.$ test). (C) Gene set enrichment analysis was performed using gene expression profiles obtained by RNAseq after exposure to $\mathrm{FX} 1$ as compared with vehicle in the indicated cell lines, against the ranked list of genes induced by BCL6 siRNA in DLBCL cells. Bars in A and B represent mean \pm SEM of 3 independent experiments. NES, normalized enrichment score. 

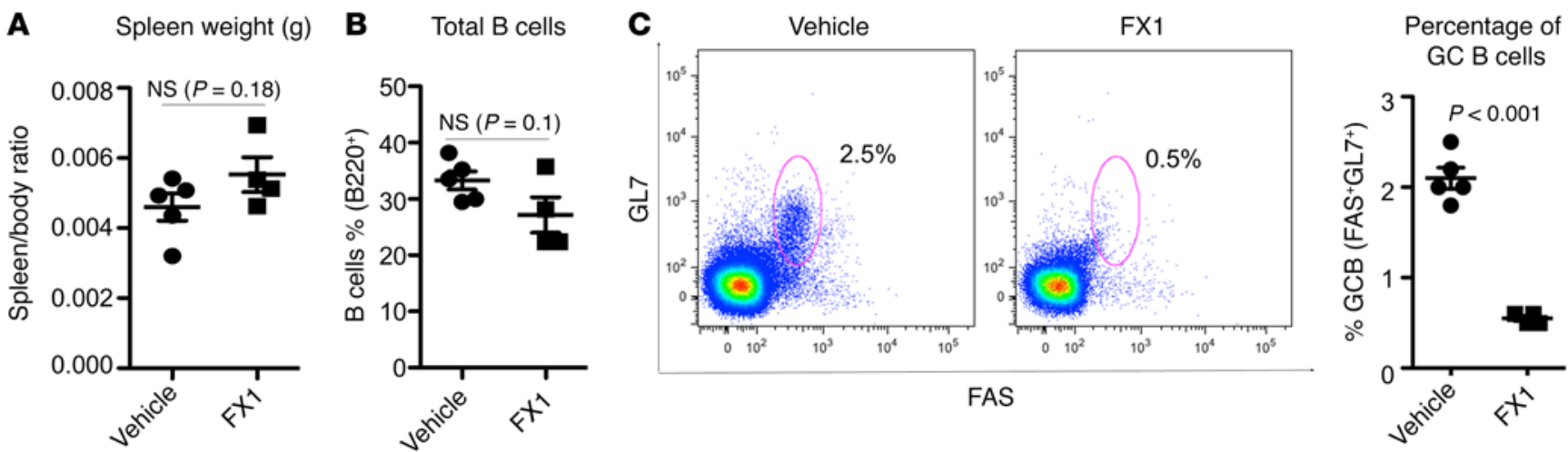

D
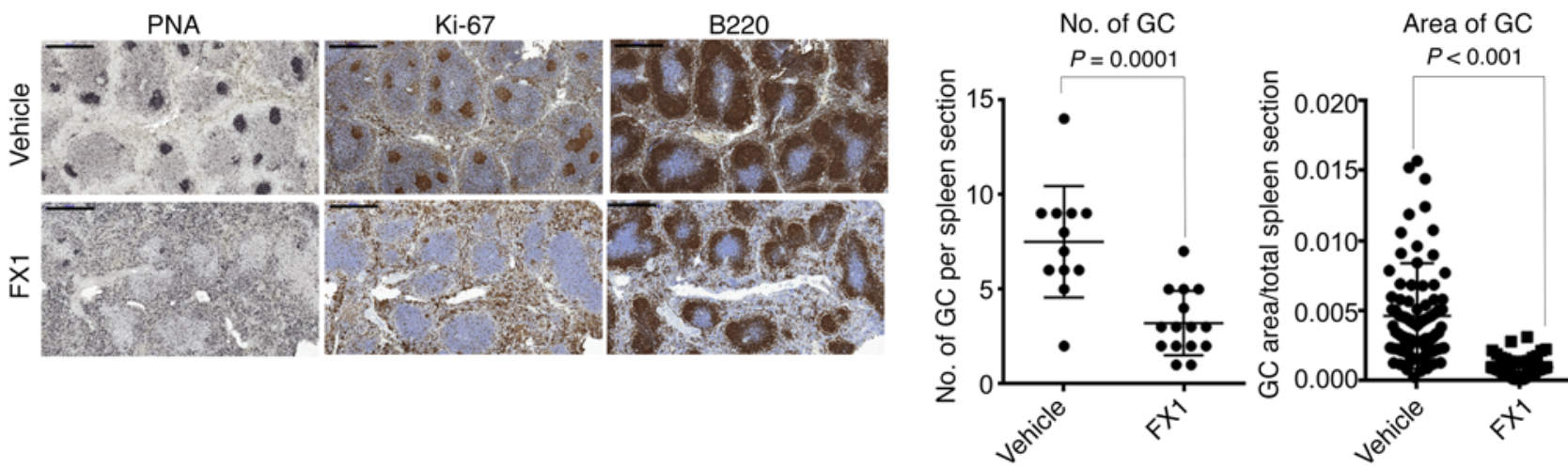

Figure 3. FX1 phenocopies the BCL6 mutant phenotype. Ten C57BL/6 mice were immunized with sheep red blood cells and then treated i.p. with 80 mg/ $\mathrm{kg} / \mathrm{d}$ FX1 or vehicle alone daily for 8 days starting 48 hours after immunization. (A) Spleen weights from mice treated with FX1 or vehicle (2-tailed MannWhitney unpaired test). (B) Flow cytometry quantification of total B cells (B220+) ( $t$ test). (C) Flow cytometry quantification of GC B cells (B220+DAPI $\mathrm{GL7}^{+} \mathrm{FAS}^{+}, t$ test). (D) IHC of spleens from mice treated with FX1 or vehicle and stained with peanut agglutinin (PNA), Ki-67, and B220. Scale bars: $500 \mu \mathrm{m}$. Quantification of number and area of GCs was performed by Imagej software. The $y$ axis shows number of positive cells/total cell number of different sections of spleens $(n=10)$ (2-tailed Mann-Whitney unpaired test). Values in $\mathbf{A}-\mathbf{D}$ are shown as mean \pm SEM.

inhibits $80 \%$ of BCL6 activity $(10 \mu \mathrm{M})$ (Supplemental Figure 1C and Supplemental Table 1). FX1 failed to significantly inhibit of any of these kinases.

To more precisely characterize the interaction between FX1 and BCL6, we next performed NMR studies of the inhibitorprotein complex. The presence of FX1 caused multiple chemical shift perturbations in the BTB domain (Figure 1G). Chemical shift perturbations reflect changes in the environment of the backbone amide upon ligand binding (31). Analysis of the residues undergoing perturbations showed them to be located in the vicinity of the lateral groove (Figure 1, G and $\mathrm{H}$ ), similar to the chemical shift perturbations observed for 79-6 (25), as suggested from the MCSILCS docking analyses. Of the perturbed residues, V49 and F61 are not on the surface of the binding site (Figure 1H). However, they are located on helices that have residues in direct contact with the ligand such that their observed chemical shift perturbations are consistent with binding in the lateral groove. Overall, the data show that FX1 binds to the BTB lateral groove with a higher affinity than SMRT, suggesting that it could be a more suitable BCL6 antagonist than 79-6.

$F X 1$ disrupts BCL 6 recruitment of corepressors to its endogenous target genes. The putative mechanism of action of BCL6 inhibitors is to disrupt formation of functional repression complexes at its target genes. To determine whether FX1 could mediate this effect, we performed quantitative ChIP assays in DLBCL cells exposed to $50 \mu \mathrm{M}$
FX1 for 30 minutes. We examined binding of BCL6 and recruitment of its corepressor proteins SMRT and BCOR to known and validated BCL6 binding sites in the CD69, CXCR4, and CDKN1A loci (32). FX1 profoundly reduced recruitment of BCOR and SMRT to all 3 BCL6 target genes, but not at a negative control locus $(P<$ 0.001; Figure 2A and Supplemental Figure 2A). In contrast, binding of BCL6 to these same sites was not impaired, and indeed was apparently increased (perhaps because of increased accessibility to antibody). In contrast, there was little presence of SMRT at these loci in the BCL6-negative DLBCL cell line Toledo, which was not affected by FX1 (Supplemental Figure 2A). The superior potency of FX1 versus 79-6 in disrupting BCL6 binding to SMRT was evident when these small molecules where compared head to head in quantitative ChIP assays in DLBCL cells after treatment with $50 \mu \mathrm{M}$ FX1 for 6 hours (Supplemental Figure 2A).

FX1 induces derepression of BCL6 target genes and transcriptional program. We next determined whether FX1 disruption of BCL6 repression complexes translated into derepression of target genes. DLBCL cells were exposed to FX1 and mRNA collected at 4 serial time points. Quantitative PCR was performed to measure the transcript abundance of the known BCL6 target genes CASP8, CD69, CXCR4, CDKN1A, and DUSP5 (Figure 2B) $(32,33)$. FX1 almost invariantly induced significant derepression of these genes as compared with vehicle in 2 independent DLBCL cell lines. Also, FX1 induced greater target gene reacti- 
A

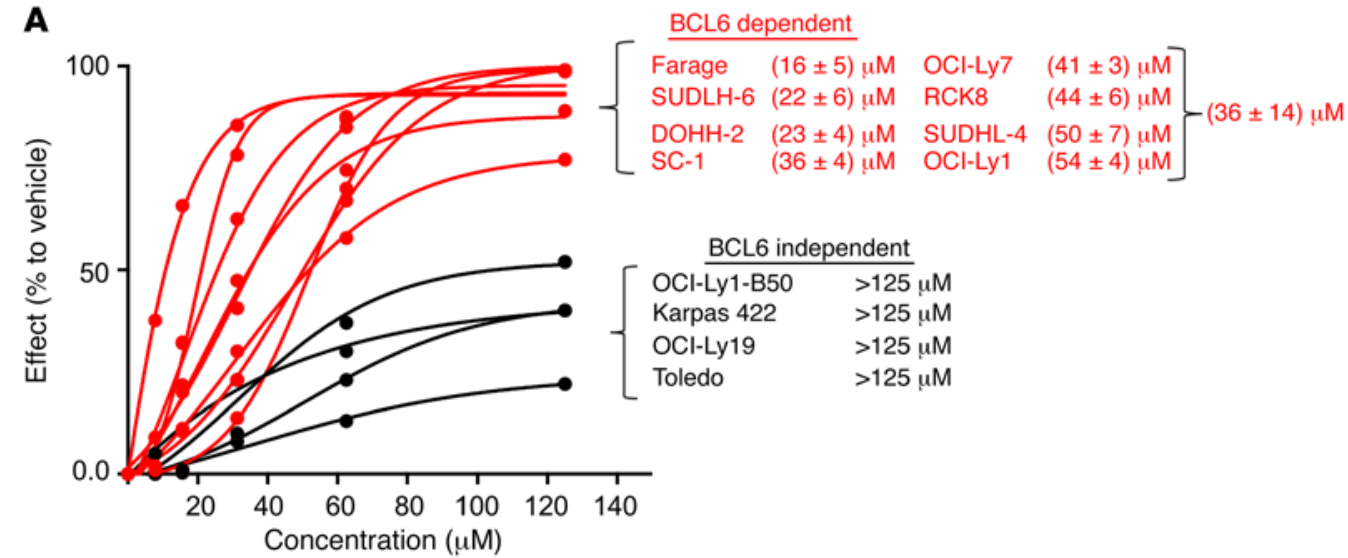

B

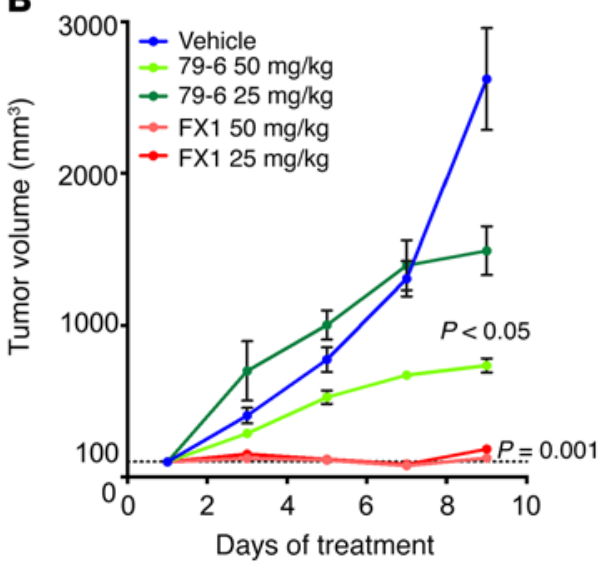

C

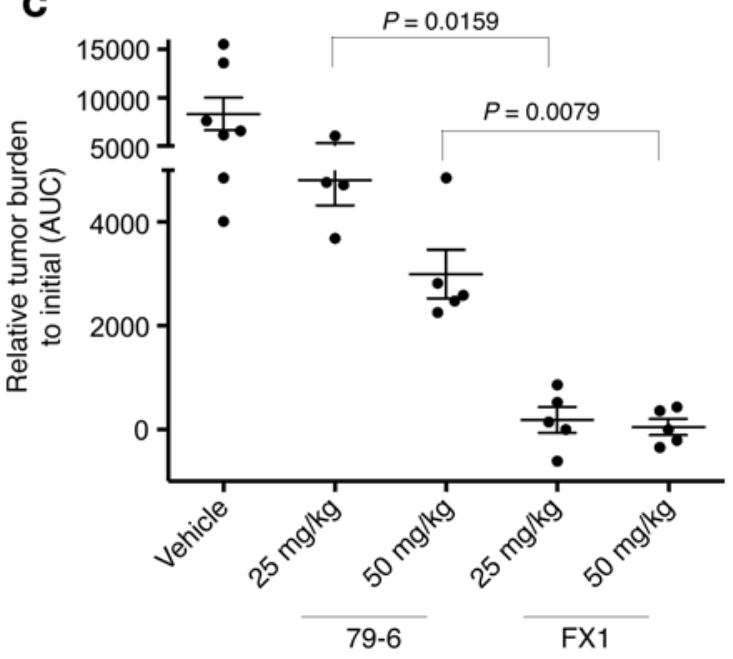

Figure 4. FX1 selectively suppresses BCL6-dependent GCBDLBCL growth in vitro and in vivo. (A) Viability of BCL6-dependent and -independent DLBCL cell lines after 48 hours of treatment with different concentrations of FX1 based on resazurin reduction. The $y$ axis shows percentage of growth-suppressing effect of the compound compared with vehicle-treated cells. Effect = $100 \%-100 \% \times$ (fluorescence of FX1-treated cells/fluorescence of vehicle-treated cells). The graph represents average of 3 independent experiments, and the $\mathrm{GI}_{50}$ values represent mean $\pm S D$ of 3 biological replicates. (B) Tumor volume of established OCI-Ly7 xenografts implanted in SCID mice treated with daily injections of 25 or $50 \mathrm{mg} / \mathrm{kg} 79-6$ or FX1 versus vehicle for 10 days $(n=10$ mice per group, 2-tailed MannWhitney unpaired test). (c) Tumor burden AUC was calculated from the same mice as in $\mathbf{B}$ between the initial tumor volume $\left(t_{0}\right.$ : $100 \mathrm{~mm}^{3}$ ) and the final volume at day 9 ( $n=10$ mice per group, 2-tailed Mann-Whitney unpaired test). Values in $\mathbf{B}$ and $\mathbf{C}$ are shown as mean $\pm \mathrm{SEM}$. vation than 79-6 (50 $\mu \mathrm{M}$, after 8 hours of incubation) in agreement with its greater disruption of BCL6 complexes (Supplemental Figure 2B).

To explore in more detail the ability of FX1 to reactivate the BCL6 transcriptional repression program, we performed RNA sequencing (RNAseq) in 3 DLBCL cell lines (SUDHL-4, SUDHL-6, and Farage) in 3 replicates after 12 hours of treatment with $50 \mu \mathrm{M}$ FX1. Between 2,000 and 3,000 genes were induced by FX1 (1.5-fold, FDR-adjusted $P<0.001)$ in individual cell lines. There were 1,027 upregulated genes in common among the 3 cell lines after treatment with FX1. We performed a gene set enrichment analysis for genes induced by BCL6 knockdown in DLBCL cells (32). We observed highly significant enrichment of FX1-upregulated genes among genes also upregulated by BCL6 siRNA (FDR < 0.001; Figure 2C). Hence, FX1 can broadly disrupt the BCL6 transcriptional program in DLBCL cells.

FX1 phenocopies the BCL6 BTB domain phenotype in vivo. Mutation of the BCL6 BTB corepressor binding site results in normal B cell development but profound loss of GC formation in mice, with only small residual GCs forming after T cell-dependent immunization (e.g., as shown in Supplemental Figure 3A and ref. 22). To determine whether FX1 could recapitulate this phenotype, we immunized C57BL/6 mice with sheep red blood cells, a $\mathrm{T}$ cell-dependent antigen, and then treated them with daily doses of FX1 at $80 \mathrm{mg} / \mathrm{kg}$ or vehicle. After 10 days of treatment (when GCs are normally at their peak), mice were euthanized and spleens were collected. Spleens in FX1-treated mice were mac- roscopically indistinguishable from vehicle controls (Figure 3A). As expected, total B cell abundance measured by flow cytometry was unaffected by FX1 (Figure $3 \mathrm{~B}$ ). In contrast and similar to the BCL6 BTB mutant phenotype, GC B cells $\left(\mathrm{GL}^{+} \mathrm{FAS}^{+} \mathrm{B} 22 \mathrm{O}^{+}\right)$ were significantly depleted by exposure to FX1 $(P<0.0001$; Figure 3C). We also examined splenic architecture by IHC. Staining with B220 antibody revealed normal B cell follicular structures, whereas staining for the GC B cell-specific marker peanut agglutinin showed profound loss of GCs (Figure 3D). This defect was further manifest by the significant reduction in the number of GCs $(P=0.0001)$ and the spleen surface area occupied by GCs (2.4-fold; $P<0.001$ ) in comparison with vehicle control (Figure $3 \mathrm{D})$. Loss of proliferating GC B cells was also evident through Ki-67 staining, which showed loss of $\mathrm{Ki}-67^{+}$GC structures in FX1versus vehicle-treated mice (Figure 3D).

FX1 potently suppresses BCL6-dependent GCB-DLBCLs in vitro and in vivo. The purpose of FX1 is to kill BCL6-dependent tumors. BCL6 is highly expressed in the GCB class of DLBCLs. DLBCL cell lines have been previously classified as BCL6 dependent or independent on the basis of whether they are affected by BCL6 knockdown or inhibition (34). To assess the capacity of FX1 to suppress DLBCLs, we treated a panel of GCB-DLBCL cell lines (8 BCL6 dependent and 4 BCL6 independent) with different concentrations of FX1 for 48 hours and determined the concentration of compound required to inhibit $50 \%$ of growth in comparison with vehicle-treated cells $\left(\mathrm{GI}_{50}\right)$. FX1 showed a selective effect 
A
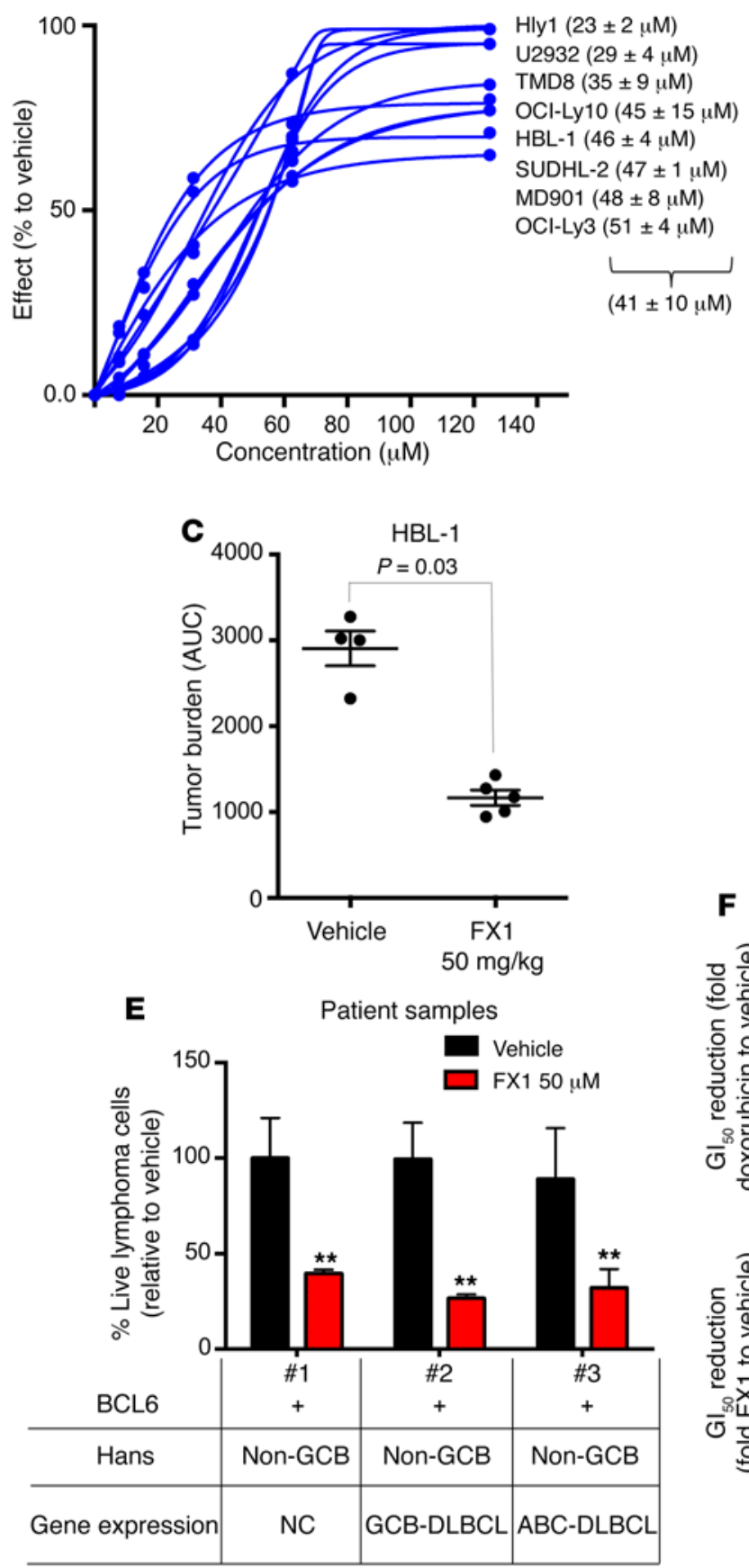

B

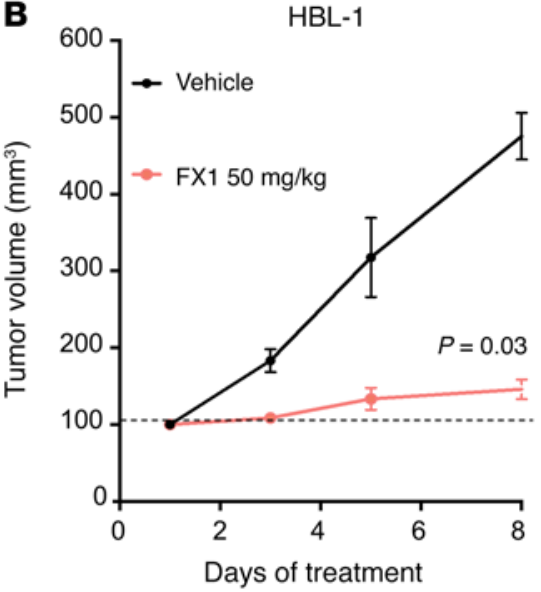

D Apoptotic nuclei (TUNEL)

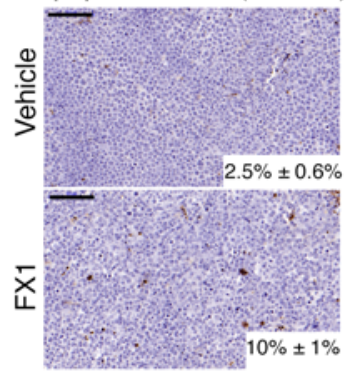

$\mathbf{F}$
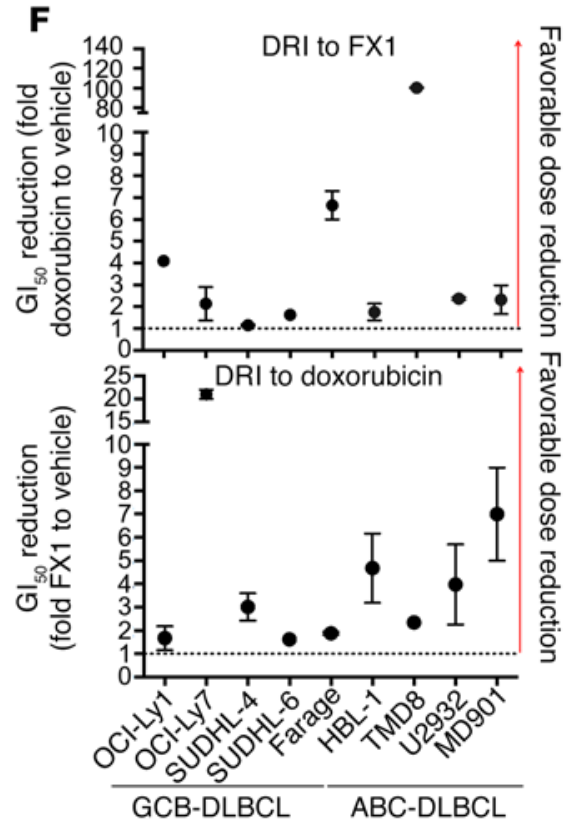

Figure 5. FX1 suppresses the growth of ABC-DLBCLs. (A) ABC-DLBCL cells were treated with increasing doses of FX1 versus vehicle. Cell viability was measured after 48 hours of treatment by resazurin reduction. The $y$ axis shows percentage of growth-suppressive effect of the compound compared with vehicle-treated cells. Effect $=100 \%-100 \% \times$ (fluorescence of FX1-treated cells/fluorescence of vehicle-treated cells). The graph represents an average of 3 independent experiments. (B) Tumor volume of established HBL-1 xenografts implanted in NOD/SCID mice during treatment with daily $50 \mathrm{mg} / \mathrm{kg}$ FX1 versus vehicle for 10 days ( $n=10$, 2-tailed Mann-Whitney unpaired test). (C) Tumor burden is shown (AUC) for the same mice as in B and calculated between the initial tumor volume $\left(t_{0}: 100 \mathrm{~mm}^{3}\right)$ and the final volume at day $9(n=10,2$-tailed Mann-Whitney unpaired test). (D) TUNEL IHC was performed in the same mice as in B. Scale bars: $100 \mu \mathrm{m}$. (E) Three primary DLBCL specimens were maintained in a coculture system and treated with FX150 $\mu \mathrm{M}$ versus vehicle. The $y$ axis represents viability of 2 biological replicates (represented as percentage of vehicle-treated cells) determined using annexin V/DAPI flow cytometry after 48 hours. Live cells are defined as CD20+CD3- cells that are annexin V/DAPI double negative (unpaired $t$ test, ${ }^{* *} P<0.005$ ). Hans and gene expression classification is shown below each sample number. (F) Combinatorial dosing of FX1 and doxorubicin is shown in the indicated cell lines, in cells treated with both agents simultaneously. The $y$ axis represents the dose-reduction index (DRI) of 3 independent experiments, based on the DRI for both doxorubicin and FX1. (DRI > 1 = favorable combination.) Values in $\mathbf{A}$ and $\mathbf{D}$ represent mean \pm SD. Values in $\mathbf{B}, \mathbf{C}, \mathbf{E}$, and $\mathbf{F}$ are shown as mean \pm SEM. 
on BCL6-dependent DLBCLs with average $\mathrm{GI}_{50}$ values of about $36 \mu \mathrm{M}$, whereas $\mathrm{GI}_{50}$ values could not be determined in BCL6independent DLBCLs, since they did not even reach $50 \%$ growth inhibition at concentrations of drug higher than $125 \mu \mathrm{M}$ (Figure $4 \mathrm{~A})$. It should be noted that some of the DLBCL cell lines defined as BCL6 independent still retain a small degree of responsiveness to BCL6 inhibitors (34). The mechanism through which loss of BCL6 dependency occurs is not known, although in certain cases (e.g., Toledo cells) this is accompanied by almost complete loss of BCL6 expression (Supplemental Figure 4A).

We next wished to assess the antilymphoma activity of FX1 in vivo. However, we first assessed the basic pharmacokinetics of the compound. Sixteen SCID mice bearing SUDHL-6 xenografts were given $50 \mathrm{mg} / \mathrm{kg}$ of FX1 via i.p. injection, followed by serial collection of serum and tissue specimens. FX1 was observed to maintain a serum concentration of $50 \mu \mathrm{M}$ for 10 hours (Supplemental Figure $4 \mathrm{~B}$ ). The half-life was estimated to be approximately 12 hours (Supplemental Figure 4B). mRNA extracted from tumor tissue from these mice was assessed for abundance of BCL6 target genes CD69, CDKN1A, and CXCR4. Maximal induction of target genes occurred at 4-6 hours and subsequently declined (Supplemental Figure 4C). Finally, we assessed whether FX1 could induce toxic effects in mice. BCL6 BTB mutant mice have no organ dysfunction or illness, and so we would not expect on-target toxicity (22). A cohort of $10 \mathrm{BALB} / \mathrm{c}$ mice were treated for 10 days with daily doses of $100 \mathrm{mg} / \mathrm{kg} / \mathrm{d} \mathrm{FX1}$, after which the animals were sacrificed and analyzed for histological evidence of organ damage (Supplemental Figure 4D and Supplemental Table 2). No signs of toxicity, inflammation, or infection were evident from H\&E-stained sections of lung, gastrointestinal tract, heart, kidney, liver, spleen, and bone marrow of the fixed organs from mice treated with FX1 compared with vehicle. We also examined peripheral blood counts and serum chemistry in FX1-treated mice, all of which remained within normal parameters (Supplemental Table 2).

To determine efficacy of FX1 as compared with 79-6 in vivo, we first established DLBCL xenografts using OCI-Ly7 DLBCL cells in SCID mice. When tumors reached a volume of approximately $100 \mathrm{~mm}^{3}$, treatment was initiated with $25 \mathrm{mg} / \mathrm{kg}$ or 50 $\mathrm{mg} / \mathrm{kg}$ of FX1 or 79-6 daily, or vehicle. Animals were sacrificed when vehicle controls reached maximal permitted tumor burden (at day 10). Strikingly, FX1 caused profound and significant suppression of DLBCLs $(P=0.001$; Figure $4 \mathrm{~B})$, and indeed not only prevented growth of the xenografts but in addition caused these tumors to shrink from their initial volume. The maximal effect was already achieved by the lower $25 \mathrm{mg} / \mathrm{kg}$ dose. In contrast, 79-6 exhibited much weaker antitumor activity at these doses (42\% reduction in tumor growth at $25 \mathrm{mg} / \mathrm{kg}$ and $64 \%$ with 50 $\mathrm{mg} / \mathrm{kg}$ ) (Figure $4 \mathrm{C}$ ). TUNEL and Ki-67 staining showed that FX1 also induced more apoptosis and growth arrest than 79-6, respectively (Supplemental Figure 4E). This potent dose-dependent antilymphoma effect of FX1 was confirmed in a second xenograft model using SUDHL-6 DLBCL cells (Supplemental Figure 4, F and $G$ ). To confirm that antitumor effects were lymphoma cell autonomous and not due to effects on the host, we also tested the effect of FX1 on BCL6-independent Toledo xenografts. Exposure to $50 \mathrm{mg} / \mathrm{kg} \mathrm{FX1}$ had no effect on these xenografts (Supplemental Figure 4, H-J).
$A B C-D L B C L$ s are biologically dependent on BCL6 and can be targeted by FX1. ABC-DLBCLs are associated with unfavorable clinical outcome and relative resistance to $\mathrm{CHOP}$ plus rituximab (RCHOP) (5). ABC-DLBCLs feature BCL6 translocations and amplifications, and yet, because BCL6 expression is usually (but not always) lower than in GCB-DLBCLs, they are generally thought of as being BCL6 independent, although this has never been formally tested. We performed Western blots in 8 ABC-DLBCL cell lines (HBL-1, TMD8, OCI-Ly3, OCI-Ly10, MD901, U2932, Hly1, and SUDHL-2) and confirmed the presence of BCL6 protein (Supplemental Figure 5A). To determine whether ABC-DLBCLs are biologically dependent on BCL6, we evaluated the growth inhibition capacity of FX1 on these 8 ABC-DLBCL cell lines. All of the ABC-DLBCL cells were about as sensitive as GCB-DLBCL cells to FX1, with an average $\mathrm{IC}_{50}$ of $41 \mu \mathrm{M}$ (Figure 5A). To verify that these cell lines are BCL6 dependent using an independent method of perturbing BCL6, we transduced 1 GCB-DLBCL and 3 ABC-DLBCL cell lines with lentivirus expressing 2 different BCL6 shRNA, as well as a nontargeted control shRNA. BCL6 shRNA 1 exhibited moderate BCL6 knockdown, whereas knockdown by shRNA 2 was more complete (Supplemental Figure 5, B and C). Compared with the control vector, both the GCB- and the ABCDLBCL cell lines exhibited reduced viability in a dose-dependent manner after transduction with the respective BCL6 shRNA lentiviruses (Supplemental Figure 5, B and C). Hence, BCL6 is required to maintain the growth of both GCB- and ABC-type DLBCLs.

To verify that ABC-DLBCLs can also be targeted by FX1 in vivo, we generated xenografts using the HBL-1 ABC-DLBCL cell line suspended in Matrigel s.c. in NOD/SCID mice. When tumors reached about $100 \mathrm{~mm}^{3}$, we treated the mice with daily doses of $50 \mathrm{mg} / \mathrm{kg} \mathrm{FX1}$. After 10 days of treatment we observed an approximately $70 \%$ decrease in the tumor volume of mice exposed to 50 $\mathrm{mg} / \mathrm{kg} \mathrm{FX1}$ (Figure 5, B and C). We also observed an increase in the apoptotic cells from $2.5 \%$ to $10 \%$ by TUNEL staining in FX1-treated tumors compared with those treated with vehicle (Figure 5D).

Primary human DLBCLs may behave differently than cell lines. We therefore generated single-cell suspensions of 3 viable primary DLBCL samples classified as being of the non-GCB subtype by Hans IHC criteria (35). All samples expressed BCL6 (Figure 5E and Supplemental Table 3). These primary DLBCLs were plated on an HK feeder layer for short-term culture and then exposed to $50 \mu \mathrm{M}$ of FX1 or vehicle for 48 hours. The fraction of live cells was then determined by annexin V/DAPI flow cytometry. As shown in Figure 5E, treatment with a single dose of $50 \mu \mathrm{M}$ of FX1 for 48 hours significantly decreased the survival of all 3 non-GCB primary samples compared with vehicle control. Because Hans criteria do not always accurately reflect DLBCL classification as determined by gene expression profiling, we also performed RNAseq on all 3 specimens and applied a cell of origin classifier to determine their subtype (36). One sample was confirmed as ABC-DLBCL, another was unclassifiable, and the third was GCB-DLBCL (Figure 5E and Supplemental Table 3). Hence these data underline that BCL6 inhibitors may be active in DLBCL regardless of cell or origin classification.

Since BCL6 inhibitors are likely to be tested clinically in combination with chemotherapy, we tested whether FX1 could enhance the response of DLBCL cells to doxorubicin, which is 
part of the standard RCHOP regimen $(6,8)$. Five GCB- and 4 ABC-DLBCL cell lines were treated with increasing doses of FX1 alone or in combination with doxorubicin. To determine whether any favorable effect might be related to how dosing is scheduled, we tested different options: FX1 and doxorubicin simultaneously, pretreatment with FX1 for the first 24 hours followed by doxorubicin for 48 hours, or the reciprocal experiment pretreating with doxorubicin followed by FX1. The simultaneous combination of doxorubicin with FX1 decreased doxorubicin $\mathrm{GI}_{50}$ 1.5- to 100-fold in the various tested cell lines (Figure 5F). The best scheme of combination was obtained when FX1 was added 24 hours after treatment with doxorubicin or simultaneously, even though adding FX1 before was also beneficial (Figure 5F and Supplemental Figure 5, D and E). Therefore, FX1 enhances response to doxorubicin in GCB-DLBCLs as well as the more chemotherapy-resistant ABC-DLBCLs.

\section{Discussion}

BCL6 is required for the survival of lymphomas and other tumors and hence is a therapeutic target of great interest (37). Transcription factors like BCL6 generally mediate their actions through protein-protein interactions rather than through enzymatic functions. Disruption of protein interactions with small molecules can be quite challenging given the size and complexity of the interface between many proteins (38). Accordingly, to date, all available inhibitors disrupt BCL6 protein interactions at concentrations higher than the critical threshold of endogenous BCL6-corepressor binding $(24,25)$. Fortunately, the BCL6 BTB domain binds to corepressors with relatively low affinity $\left(20-30 \mu \mathrm{M} K_{\mathrm{D}}\right)(20)$. Hence, even though protein interactions are difficult to target, it is not necessary to generate super-high-affinity compounds, since the inhibitors only need to compete against a relatively weak protein interface in the BCL6 BTB domain.

Herein we used a sophisticated in silico functional group-based screening approach called SILCS $(26,27)$ to design improved small molecules geared to bind the lateral groove motif of the BCL6 BTB domain and disrupt binding of endogenous corepressors. SILCS mapping of the BCL6 BTB domain lateral groove provided crucial information to guide medicinal chemistry refinement of BCL6 small-molecule design. Among these crucial observations were the important contributions of the indoline-2-one ring along with its halogen substituent to binding as well as the need for only a single carboxylate moiety, versus the 2 carboxylates on the lead compound 79-6. These observations guided the optimization of improved BCL6 inhibitors based on 79-6, most notably FX1, that cross the critical threshold of manifesting a greater binding affinity for BCL6 than endogenous corepressors. FX1 was more than 100-fold more powerful than the previous generation of BCL6 inhibitors represented by 79-6, and 300-fold more potent than the recently reported binding of the antibiotics rifamycin and rifabutin $\left(K_{\mathrm{D}} \sim 1 \mathrm{mM}\right)$ (39). Importantly, this superior potency in binding translated to improved efficacy in cell-based assays and animal models of GCB-DLBCL, while not leading to toxicity. Moreover, improved potency was achieved without sacrificing specificity, since FX1 had no effect on other BTB proteins either in the context of binding purified BTB domains or in reporter assays informing transcription repressor activity. This specificity is likely due to the fact that the surface residues that line the BTB lateral groove and bind to corepressors are unique to BCL6 and not conserved in other BTB proteins (20). Hence this SILCS approach can successfully guide the design of protein-protein interaction inhibitors.

Development of improved BCL6 inhibitors enabled us to probe an important question in lymphoma biology and experimental therapeutics related to the relative significance of BCL6 function in ABC-DLBCLs. This class of DLBCLs is thought to reflect $B$ cells that are exiting the $G C$ and transitioning to plasma cells. The fact that ABC-DLBCLs often have lower levels of BCL6 than GCB-DLBCLs has created an impression that BCL6 might not be important in these tumors. Yet tumor genetics suggests otherwise, since $\mathrm{ABC}-\mathrm{DLBCLs}$ feature translocations that induce constitutive expression of BCL6 as well as amplifications of BCL6 locus $(10,12)$. It has been suggested that BCL6 might function through a hit-and-run-type mechanism in ABC-DLBCLs on the basis of studies where transient expression of BCL6 in murine stem cells induced DLBCLs later on in these mice (12). Our data using shRNA and FX1 in DLBCL cell lines in vitro and in vivo, as well as using FX1 in primary human ABC-DLBCL cells, reveal that BCL6 is indeed biologically active and required by these tumors to maintain their growth and survival. Yet it is still possible that BCL6 hit-and-run effects might sometimes occur in DLBCL, as a subset of BCL6-independent DLBCL cells still express BCL6 though are no longer dependent on it (34).

In many cell lines it appears that response to BCL6 inhibitors correlates with BCL6 levels, in that higher doses are required to suppress cell lines that express more BCL6. For example, OCI-Ly1 cells express high levels of BCL6 and need more compound than SUDHL-6 and DOHH-2 cells, which express less BCL6. However, there are many exceptions to this rule, likely due to the effects of different sets of somatic mutations and other factors that may alter BCL6 functionality. The study of primary DLBCLs is limited by the fact that these tumors cannot be maintained in culture long enough to fully assess the therapeutic impact of FX1. Yet our studies in primary specimens serve as proof of principle confirming cell line data showing that GCB- and ABC-DLBCL cells are BCL6 dependent and amenable to BCL6 inhibitors. Finally, FX1 could enhance the response of both GCB- and ABC-DLBCLs to cytotoxic therapy. It is therefore possible that combination of BCL6-targeted therapy with standard antilymphoma drugs could yield superior clinical efficacy, which would be of great interest especially in higher-risk patients who are more likely to be chemotherapy resistant. Hence our study expands the scope of lymphomas likely to respond to BCL6-targeted therapy to include both GCB- and ABC-type DLBCLs. This is particularly important given the more unfavorable outcome of ABC-DLBCLs. Efforts to further refine FX1 and generate additional chemical scaffolds using SILCS will be expected to yield compounds that could benefit these difficult-to-treat patients.

\section{Methods}

Supplemental Methods are available online with this article; doi:10.1172/JCI85795DS1.

Chemicals. 79-6 was purchased from Millipore, and the SMRT peptide (amino acids 1414-1430 corresponding to the SMRT BCL6 binding domain; $\mathrm{NH}_{2}$-LVATVKEAGRSIHEIPR-COOH; MW 1,876.21 
$\mathrm{g} / \mathrm{mol}$ ) was from Bio-Synthesis Inc. Recombinant BCL6 BTB domain fragment (amino acids 1-129) was produced at the Protein Production Facility of Cornell University as previously described (20).

Cell lines. Human embryonic kidney 293T cells, and human DLBCL cell lines Toledo, Farage, SUDHL-2, SUDHL-4, SUDHL-6, and SC-1, were purchased from ATCC. Human DLBCL cell lines OCI-Ly1, OCI-Ly3, OCI-Ly7, OCI-Ly10, OCI-Ly19, DOHH-2, RCK8, and Karpas 422 were from the DSMZ German collection of microorganisms and cell cultures. OCI-Ly1-B50 are OCI-Ly1 cells that were grown in the presence of increasing concentrations of RI-BPI and grow independently of BCL6 inhibitors. DLBCL cell lines TMD8, HBL-1, Hly1, U2932, and MD901 were provided by Jose Angel Martinez-Climent, Centre for Applied Medical Research (CIMA), Pamplona, Spain.

All human cell lines were identified and authenticated by DNA genotyping before use. DNA extraction, short tandem repeat profiling, and comparison with known cell line profiles from ATCC were performed by Bio-Synthesis Inc. 293T cells were grown in DMEM supplemented with 10\% FBS (Invitrogen) and 1\% penicillin-streptomycin (Invitrogen). DLBCL cell lines OCI-Ly1, OCI-Ly1-B50, OCI-Ly3, OCI-Ly7, OCI-Ly10, and OCI-Ly19 were grown in Iscove's medium (Invitrogen), 10\% FBS, and 1\% penicillin-streptomycin. DLBCL cell lines Toledo, Farage, SUDHL-4, DOHH-2, SC-1, RCK8, TMD8, HBL-1, Karpas 422, Hly1, U2932, SUDHL-2, MD901, and SUDHL-6 were cultured in 90\% RPMI medium (Invitrogen), 10\% FBS, 2 mM glutamine, $10 \mathrm{mM}$ HEPES (Invitrogen), and 1\% penicillin-streptomycin. All cell lines were cultured at $37^{\circ} \mathrm{C}$ in a humidified atmosphere of $5 \% \mathrm{CO}_{2}$.

MST measurements. Recombinant BCL6 BTB was labeled using the RED-NHS Labeling kit (NanoTemper Technologies $\mathrm{GmbH}$ ). The labeling reaction was performed according to the manufacturer's instructions in the supplied labeling buffer applying a concentration of $20 \mu \mathrm{M}$ protein (molar dye/protein ratio $2: 1$ ) at room temperature for 30 minutes. Unreacted dye was removed with the supplied dye removal columns equilibrated with PBS buffer (PBS, 0.005\% Tween-80) (Invitrogen). The label/protein ratio was determined using photometry at $650 \mathrm{~nm}$ and Bradford reagent (Invitrogen). Thereby, a ratio of 0.8 was typically achieved. The labeled BCL6 BTB was adjusted to 400 nM with PBS buffer supplemented with $0.05 \%$ Tween-80. SMRT, 79-6, and FX1 were dissolved in PBS buffer supplemented with $0.05 \%$ Tween-80 and 10\% DMSO, and a series of 16 1:1 dilutions were prepared in the identical buffer, producing ligand concentrations ranging from $19 \mathrm{pM}$ to $625 \mu \mathrm{M}$. For thermophoresis, each ligand dilution was mixed with 1 volume of labeled BCL6 BTB, which leads to a final concentration of fluorescently labeled BCL6 BTB of $200 \mathrm{nM}$ and final ligand concentrations ranging from $9 \mathrm{pM}$ to $312 \mathrm{nM}$ in a $5 \%$ DMSO final concentration. After 10 minutes of incubation, approximately $4 \mu \mathrm{l}$ of each solution was filled into Monolith NT Standard Treated Capillaries (NanoTemper Technologies $\mathrm{GmbH}$ ). Thermophoresis was measured using a Monolith NT.115 instrument (NanoTemper Technologies $\mathrm{GmbH}$ ) at a temperature of $25^{\circ} \mathrm{C}$ with 5 seconds $/ 30$ seconds $/ 5$ seconds laser off/on/off times, respectively. Instrument parameters were adjusted with $90 \%$ light-emitting diode (LED) power and $40 \%$ MST power. Data of 3 independent experiments were analyzed (NT Analysis software version 1.5.41, NanoTemper Technologies $\mathrm{GmbH}$ ) using the signal from thermophoresis.

Purification of BCL6 BTB domain. BCL6 BTB domain was expressed in E. coli BL21 (DE3) cells as fusion with thioredoxin. Pro- tein was purified using Ni-NTA affinity chromatography (Invitrogen) and cleaved with thrombin protease (Sigma-Aldrich). At the final step, protein was purified using size exclusion chromatography with Superdex 75 column (Sigma-Aldrich) into crystallization buffer (50 $\mathrm{mM}$ Tris- $\mathrm{HCl}, \mathrm{pH} 7.5,150 \mathrm{mM} \mathrm{NaCl}$, and $1 \mathrm{mM}$ tris[2-carboxyethyl] phosphine [TCEP]). For NMR experiments, $15 \mathrm{~N}$ labeled protein was purified using identical protocol and was exchanged to $50 \mathrm{mM}$ Tris, pH7.5, $150 \mathrm{mM} \mathrm{NaCl}$, and $1 \mathrm{mM}$ TCEP buffer.

NMR experiments. All NMR experiments were recorded at $30^{\circ} \mathrm{C}$ using a Bruker $600 \mathrm{MHz}$ spectrometer equipped with cryogenic probe (Bruker Corp.). Assignment of BCL6 BTB domain was performed based on HNCA and HNCOCA experiments recorded for $200 \mu \mathrm{M} 13 \mathrm{C}, 15 \mathrm{~N}$ BCL6, and previously published assignment (25). Fo-Fc electron density maps found in the lateral groove site of the BCL6 BTB contoured at $2.0 \sigma$ level. Due to insufficient occupancy, full structure refinement of the 1085 in the complex could not be reliably completed.

Reporter assays. For screening of the synthesized small molecules, we transfected $5 \times 10^{5} 293 \mathrm{~T}$ cells in a 6-well plate using polyethylenimine (Polysciences Inc.) with a BCL6 BTB luciferase reporter assay, as previously reported $(23,25)$. Alternatively, cells were transfected with 1,320 ng of plasmid containing the Kaiso-BTB domain fused to GAL4DBD, 500 ng HIC-BTB-GAL4-DBD (23), 500 ng PLZF-BTB-GAL4DBD (23), or GAL4-DBD alone as shown previously (25).

Determination of growth inhibition. DLBCL cell lines were grown in exponential growth conditions over a 48-hour drug exposure time. Cell viability was determined with the fluorescent redox dye CellTiter-Blue (Promega). Fluorescence was determined for 3 replicates per treatment condition or vehicle with the Synergy NEO microplate reader (BioTek). Cell viability of the drug-treated cells was normalized to their vehicle-treated controls, and the results were expressed as percentage viability. We calculated the drug effect as 100 - percentage viability. Through dose-effect curves we determined the drug concentration that inhibits the growth of cell lines by $50 \%$ compared with vehicle $\left(\mathrm{GI}_{50}\right)$ with CompuSyn software (Biosoft). Experiments were performed in triplicate.

Combination of FX1 with doxorubicin. For combination treatments, we exposed cells to a dose curve of each drug alone or their combination in constant ratio, and cell viability was determined by CellTiterBlue (Promega). To compare different schedules of treatments, we treated the cells in triplicate as follows: FX1 and doxorubicin simultaneously and cells treated for 48 hours; FX1 first and 24 hours after we added doxorubicin and treated for an extra 48 hours; doxorubicin first and 24 hours after we added FX1 and treated for an extra 48 hours. Then, we used CompuSyn software (Biosoft) to plot dose-effect curves and calculate the dose-reduction index.

Mouse xenograft studies. Six- to 8-week-old male SCID mice (Jackson Laboratory) were injected s.c. with $10^{7}$ low-passage human SUDHL-6, OCI-Ly7, or Toledo cells. Alternatively, 6- to 8-week-old NOD/SCID mice (Jackson Laboratory) were injected with low-passage HBL-1 cells. When tumors reached a palpable size (approximately $100 \mathrm{~mm}^{3}$ ), mice were assigned in a randomized way to treatment groups and treated i.p. with 25 or $50 \mathrm{mg} / \mathrm{kg} / \mathrm{d}$ of the drugs. Drugs were reconstituted in PEG- 400 and stored at $-20^{\circ} \mathrm{C}$ until use. Tumor size was measured 3 times a week with an electronic digital caliper (Invitrogen) in 2 dimensions, and then tumor volume was calculated with the formula: tumor volume $\left(\mathrm{mm}^{3}\right)=\left(\right.$ smallest diameter ${ }^{2}$ 
$\times$ largest diameter) $/ 2$. Data are expressed as mean \pm SEM, and differences were considered statistically significant at $P$ less than 0.05 by Mann-Whitney test.

Primary cell treatment. Single-cell suspensions from lymph node biopsies were thawed and resuspended in Advanced RPMI supplemented with 20\% human serum, GlutaMAX (2X) (Invitrogen), glycine $(5 \mathrm{mM})$, and penicillin and streptomycin. Cell number and viability were determined by counting with trypan blue (catalog SV3008401; Thermo Fisher). Irradiated HK cells (2,000 rad) cultured in DMEM supplemented with $10 \% \mathrm{FBS}$ and penicillin and streptomycin adhered to tissue culture plates at $37^{\circ} \mathrm{C}$. Medium was aspirated and patient samples were plated on the HK feeder layer. Samples were exposed to FX1 or vehicle for 48 hours and analyzed for viability by staining with PerCP Cy5.5-conjugated anti-CD3 (340948; BD Biosciences) and FITC-conjugated anti-CD2O (347673; BD Biosciences) as well as annexin $\mathrm{V}$ and DAPI. $\mathrm{CD}^{2} \mathrm{O}^{+} \mathrm{CD}^{-}$cells that were annexin V/DAPI double negative were considered live, malignant B cells. Primary samples were classified by Hans IHC criteria (35).

Statistics. We used Student's $t$ test for statistical analysis, and then the analysis was performed with the software GraphPad Prism 6. $P$ less than 0.05 was considered as significant. For animal experiments, we used 2-tailed unpaired $t$ test unless otherwise stated. Specifically, 2-tailed Mann-Whitney unpaired test was used for xenograft growth experiment analysis. All statistical analyses were carried out using Prism software (GraphPad Software).

Study approval. All procedures involving animals followed $\mathrm{NIH}$ protocols and were approved by the Animal Institute Committee of the Weill Cornell Medical College of Cornell University. We obtained deidentified human tissues in accordance with and with approval from the IRB of the New York Presbyterian Hospital. Human ABC-DLBCL samples were obtained in accordance with and with approval from the IRB of the New York Presbyterian Hospital.

Accession numbers. The Gene Expression Omnibus accession numbers for the expression RNAseq data reported in this paper are GSE79888 and GSE80351.

\section{Author contributions}

MGC designed, conducted, and performed experiments; acquired and analyzed data; discussed results; and wrote the manuscript. WY did SILCS simulations and predictions of interactions. WB conducted experiments, acquired and analyzed data, and discussed results. MRT and HG analyzed data and discussed results. RLG designed, conducted, and performed experiments; acquired and analyzed data; and discussed results. EO performed experiments, acquired and analyzed data, and discussed results. $\mathrm{KH}$ conducted experiments, acquired and analyzed data, and discussed results. SNY performed experiments and acquired data. JC performed experiments and acquired and analyzed data. RS analyzed data and discussed results. KV did SILCS simulations and predictions of interactions. HC and DL synthesized compounds. HJC and JA did NMR and crystallography studies. WT and JPL obtained patient samples. WD and OE designed and analyzed the ABC-DLBCL patient sample classification. LC designed experiments and discussed results. TC, FX, ADM, and AMM designed experiments, discussed results, and wrote the paper.

\section{Acknowledgments}

This work was supported by the Leukemia and Lymphoma Society Therapy Acceleration Program. We thank all the people from the Melnick and MacKerell laboratories for discussion and suggestions; Christine Crosby and all the people from NanoTemper Technologies $\mathrm{GmbH}$ for helping with the setup and discussion of the results of the MST; and Carolina Adura, Antonio Luz, Jeanne Chiaravalli Giganti, and Fraser Glickman from the HighThroughput and Spectroscopy Resource Center at Rockefeller University for all their help and suggestions about $K_{\mathrm{D}}$ determination. We also thank all the people from the Epigenomics Core at Weill Cornell Medicine for helping us with the RNAseq processing. We also thank Shilei Zhu for helping us with the evaluation of the solubility of the small molecules. Appreciation to the Samuel Waxman Cancer Research Foundation for financial support and the University of Maryland Computer-Aided Drug Design Center for computational and financial support.

Address correspondence to: Leandro Cerchietti, Department of Medicine, Weill Cornell Medical College, Cornell University, 1300 York Avenue, Meyer Research Building, C-620B, BOX 113, New York, New York 10021, USA. Phone: 212.746.7649; E-mail: lec2010@med.cornell.edu. Or to: Tomasz Cierpicki, Department of Pathology, University of Michigan, 1150 West Medical Center Drive, MSRB 1, Room 4510C, Ann Arbor, Michigan 48109, USA. Phone: 734.615.9324; E-mail: tomaszc@umich.edu. Or to: Fengtian Xue, Department of Pharmaceutical Sciences, Department of Anesthesiology, University of Maryland, Baltimore, Health Sciences Facility II, Room 637, Baltimore, Maryland 21201, USA. Phone: 410.706.8521; E-mail: fxue@rx.umaryland.edu. Or to: Alexander D. MacKerell, Department of Pharmaceutical Sciences, School of Pharmacy, University of Maryland, Baltimore, 20 Penn Street, Baltimore, Maryland 21201, USA. Phone: 410.706.7442; E-mail: amackere@rx.umaryland.edu. Or to: Ari M. Melnick, Departments of Medicine and Pharmacology, Weill Cornell Medical College, Cornell University, 413 E. 69th Street, Belfer Research Building, BB-1462, New York, New York 10021, USA. Phone: 646.962.6726; E-mail: amm2014@med.cornell.edu.
1. Evans LS, Hancock BW. Non-Hodgkin lymphoma. Lancet. 2003;362(9378):139-146.

2. Hatzi K, Melnick A. Breaking bad in the germinal center: how deregulation of BCL6 contributes to lymphomagenesis. Trends Mol Med. 2014;20(6):343-352.

3. Klein U, Dalla-Favera R. Germinal centres: role in B-cell physiology and malignancy. Nat Rev Immunol. 2008;8(1):22-33.

4. Alizadeh AA, et al. Distinct types of diffuse large
B-cell lymphoma identified by gene expression profiling. Nature. 2000;403(6769):503-511.

5. Lenz G, Staudt LM. Aggressive lymphomas. N Engl JMed. 2010;362(15):1417-1429.

6. Coiffier B, et al. CHOP chemotherapy plus rituximab compared with $\mathrm{CHOP}$ alone in elderly patients with diffuse large-B-cell lymphoma. N Engl JMed. 2002;346(4):235-242.

7. Habermann TM, et al. Rituximab-CHOP versus $\mathrm{CHOP}$ alone or with maintenance rituximab in older patients with diffuse large B-cell lymphoma. J Clin Oncol. 2006;24(19):3121-3127.

8. Pfreundschuh M, et al. CHOP-like chemotherapy with or without rituximab in young patients with good-prognosis diffuse large-B-cell lymphoma: 6-year results of an open-label randomised study of the MabThera International Trial (MInT) Group. Lancet Oncol. 2011;12(11):1013-1022.

9. Sehn LH, et al. Introduction of combined CHOP plus rituximab therapy dramatically 
improved outcome of diffuse large B-cell lymphoma in British Columbia. JClin Oncol. 2005;23(22):5027-5033.

10. Iqbal J, et al. Distinctive patterns of BCL6 molecular alterations and their functional consequences in different subgroups of diffuse large B-cell lymphoma. Leukemia. 2007;21(11):2332-2343.

11. Bea S, et al. Diffuse large B-cell lymphoma subgroups have distinct genetic profiles that influence tumor biology and improve geneexpression-based survival prediction. Blood. 2005;106(9):3183-3190.

12. Green MR, et al. Transient expression of Bcl6 is sufficient for oncogenic function and induction of mature B-cell lymphoma. Nat Commun. 2014;5:3904.

13. Ye BH, et al. The BCL- 6 proto-oncogene controls germinal-centre formation and Th2-type inflammation. Nat Genet.1997;16(2):161-170.

14. Dent AL, Shaffer AL, Yu X, Allman D, Staudt LM. Control of inflammation, cytokine expression, and germinal center formation by BCL-6. Science. 1997;276(5312):589-592.

15. Fukuda T, et al. Disruption of the Bcl6 gene results in an impaired germinal center formation. JExp Med.1997;186(3):439-448.

16. Baron BW, et al. The human BCL6 transgene promotes the development of lymphomas in the mouse. Proc Natl Acad Sci U S A. 2004;101(39):14198-14203.

17. Cattoretti G, et al. Deregulated BCL6 expression recapitulates the pathogenesis of human diffuse large B cell lymphomas in mice. Cancer Cell. 2005;7(5):445-455.

18. Huynh KD, Bardwell VJ. The BCL-6 POZ domain and other POZ domains interact with the co-repressors N-CoR and SMRT. Oncogene. 1998;17(19):2473-2484.

19. Huynh KD, Fischle W, Verdin E, Bardwell VJ. BCoR, a novel corepressor involved in BCL-6 repression. Genes Dev. 2000;14(14):1810-1823.

20. Ahmad KF, et al. Mechanism of SMRT corepressor recruitment by the BCL6 BTB domain. $M o l$ Cell. 2003;12(6):1551-1564.

21. Ghetu AF, Corcoran CM, Cerchietti L, Bardwell VJ, Melnick A, Privé GG. Structure of a BCOR corepressor peptide in complex with the BCL6 BTB domain dimer. Mol Cell. 2008;29(3):384-391.

22. Huang C, Hatzi K, Melnick A. Lineage-specific functions of Bcl-6 in immunity and inflammation are mediated by distinct biochemical mechanisms. Nat Immunol. 2013;14(4):380-388.

23. Polo JM, et al. Specific peptide interference reveals BCL6 transcriptional and oncogenic mechanisms in B-cell lymphoma cells. Nat Med. 2004;10(12):1329-1335.

24. Cerchietti LC, et al. A peptomimetic inhibitor of BCL6 with potent antilymphoma effects in vitro and in vivo. Blood. 2009;113(15):3397-3405.

25. Cerchietti LC, et al. A small-molecule inhibitor of BCL6 kills DLBCL cells in vitro and in vivo. Cancer Cell. 2010;17(4):400-411.

26. Guvench O, MacKerell AD. Computational fragment-based binding site identification by ligand competitive saturation. PLoS Comput Biol. 2009;5(7):e1000435.

27. Raman EP, Yu W, Lakkaraju SK, MacKerell AD. Inclusion of multiple fragment types in the site identification by ligand competitive saturation (SILCS) approach. JChem Inf Model. 2013;53(12):3384-3398.

28. Baell JB, Holloway GA. New substructure filters for removal of pan assay interference compounds (PAINS) from screening libraries and for their exclusion in bioassays. JMed Chem. 2010;53(7):2719-2740.

29. Seidel SA, et al. Microscale thermophoresis quantifies biomolecular interactions under previously challenging conditions. Methods. 2013;59(3):301-315.
30. Wienken CJ, Baaske P, Rothbauer U, Braun D, Duhr S. Protein-binding assays in biological liquids using microscale thermophoresis. Nat Commun. 2010;1:100.

31. Zuiderweg ER. Mapping protein-protein interactions in solution by NMR spectroscopy. Biochemistry. 2002;41(1):1-7.

32. Hatzi K, et al. A hybrid mechanism of action for BCL6 in B cells defined by formation of functionally distinct complexes at enhancers and promoters. Cell Rep. 2013;4(3):578-588.

33. Shaffer AL, Yu X, He Y, Boldrick J, Chan EP, Staudt LM. BCL-6 represses genes that function in lymphocyte differentiation, inflammation, and cell cycle control. Immunity. 2000;13(2):199-212.

34. Polo JM, et al. Transcriptional signature with differential expression of BCL6 target genes accurately identifies BCL6-dependent diffuse large B cell lymphomas. Proc Natl Acad Sci U S A. 2007;104(9):3207-3212.

35. Hans CP, et al. Confirmation of the molecular classification of diffuse large B-cell lymphoma by immunohistochemistry using a tissue microarray. Blood. 2004;103(1):275-282.

36. Wright G, Tan B, Rosenwald A, Hurt EH, Wiestner A, Staudt LM. A gene expression-based method to diagnose clinically distinct subgroups of diffuse large B cell lymphoma. Proc Natl Acad Sci U S A. 2003;100(17):9991-9996.

37. Cerchietti L, Melnick A. Targeting BCL6 in diffuse large B-cell lymphoma: what does this mean for the future treatment?. Expert Rev Hematol. 2013;6(4):343-345.

38. Wells JA, McClendon CL. Reaching for highhanging fruit in drug discovery at protein-protein interfaces. Nature. 2007;450(7172):1001-1009.

39. Evans SE, et al. The ansamycin antibiotic, rifamycin SV, inhibits BCL6 transcriptional repression and forms a complex with the BCL6-BTB/POZ domain. PLoS One. 2014;9(3):e90889. 